\title{
Polymorphism-Dependent Dynamic Ultralong Organic Phosphorescence
}

\author{
Mingxing Gu $\mathbb{D}^{1,2}$ Huifang Shi, ${ }^{2}$ Kun Ling, ${ }^{2}$ Anqi Lv, ${ }^{2}$ Kaiwei Huang, ${ }^{2}$ Manjeet Singh, \\ He Wang, ${ }^{2}$ Long Gu, ${ }^{2}$ Wei Yao, ${ }^{2}$ Zhongfu An $\oplus_{0}^{1,2}$ Huili Ma ${ }^{1,},{ }^{2}$ and Wei Huang ${ }^{1,2}$ \\ ${ }^{1}$ Institute of Flexible Electronics (IFE), Northwestern Polytechnical University (NPU), 127 West Youyi Road, Xi'an 710072, China \\ ${ }^{2}$ Key Laboratory of Flexible Electronics (KLOFE) \& Institute of Advanced Materials (IAM), Nanjing Tech University (NanjingTech), \\ 30 South Puzhu Road, Nanjing 211816, China
}

Correspondence should be addressed to Zhongfu An; iamzfan@njtech.edu.cn, Huili Ma; iamhlma@njtech.edu.cn, and Wei Huang; iamwhuang@nwpu.edu.cn

Received 17 October 2019; Accepted 24 December 2019; Published 7 February 2020

Copyright ( 2020 Mingxing Gu et al. Exclusive Licensee Science and Technology Review Publishing House. Distributed under a Creative Commons Attribution License (CC BY 4.0).

\begin{abstract}
Developing ultralong organic phosphorescence (UOP) materials with smart response to external stimuli is of great interest in photonics applications, whereas the manipulation of molecular stacking on tuning such dynamic UOP is still a formidable challenge. Herein, we have reported two polymorphs with distinct photoactivated dynamic UOP behavior based on a pyridine derivative for the first time. Our experiment revealed that the dynamic UOP behavior including photoactivation and deactivation feature is highly dependent on irradiation intensity and environmental atmosphere. Additionally, given the unique dynamic UOP feature, these phosphors have been successfully applied to phosphorescence-dependent molecular logic gate and timing data storage. This result not only paves a way to design smart functional materials but also expands the scope of the applications on organic phosphorescence materials.
\end{abstract}

\section{Introduction}

Stimuli-responsive materials, that is, smart materials, whose physical properties can be controllably tuned by external stimuli, such as heat, light, pressure, and solvent, are of typical interest because of their promising potential applications $[1,2]$. For instance, equipped with the sensibility of one or more external stimuli, the photoluminescent materials can be applied to diverse fields ranging from biological detection $[3,4]$ and sensors $[5,6]$ to optical memory devices [7-9] and logic gates $[10,11]$. Despite great potentials in practical applications, it remains a formidable challenge to develop such smart materials so far. Recently, a new type of organic luminescent materials with ultralong organic phosphorescence (UOP) has drawn considerable attentions owing to their distinctive advantages of long-lived persistent luminescence and high exciton utilization [12-15]. A library of UOP luminogens has been developed with a series of feasible strategies including crystal engineering [16-23], $\mathrm{H}$-aggregation [24-28], host-guest doping [29-32], and so on [33-44], which mainly show steady-state phosphorescence emission at room temperature. Very recently, a dynamic photoactivated UOP was fortuitously found in triazines and phenothiazine derivatives under ambient conditions [45-48]. Namely, the phosphorescence lifetime can be rationally regulated by photoactivation time. Notably, crystallization is essential to such phenomenon, implying the importance of molecular stacking on dynamic UOP. Moreover, molecular stacking has been proved to be effective in manipulating luminescent properties of organic materials in solid state [49-52]. Therefore, it is urgent to provide an insight from molecular stacking to understanding the inherent mechanism in dynamic UOP.

\section{Results}

2.1. Culture and Observation of Polymorphs. Two polymorphs of $\mathrm{PyCz}, \mathrm{PyCz}-\mathrm{B}$ (block-type crystal) and $\mathrm{PyCz}-\mathrm{N}$ (needle-like crystal), were cultured by slow solvent evaporation from different binary solvents under ambient condition. The binary solvents are ethyl acetate and $n$-hexane $\left(\mathrm{V}_{\mathrm{EA}}: \mathrm{V}_{\mathrm{Hex}}=3: 2\right)$ for PyCz-B and dichloromethane and $n$-hexane $\left(\mathrm{V}_{\mathrm{DCM}}: \mathrm{V}_{\mathrm{Hex}}=3: 2\right)$ for $\mathrm{PyCz}-\mathrm{N}$, respectively. 
The fluorescence microscopy images of these two crystalline polymorphs are depicted in Figure 1(a). Both polymorphs showed blue emission upon UV irradiation and after switching off the UV lamp under ambient conditions; no visible persistent luminescence have been observed. Impressively, both PyCz-B and PyCz-N demonstrated distinct persistent luminescence after long photoactivation for a period of time under UV light irradiation. Observationally, PyCz-B showed bright persistent luminescence that lasted for several seconds, activated by strong $365 \mathrm{~nm}$ light $\left(40 \mathrm{~mW} / \mathrm{cm}^{2}\right)$ within $3 \mathrm{~s}$, while it took much longer for the photoactivation of $\mathrm{PyCz}-$ $\mathrm{N}$, about 6 min (Figure 1(b) and Movie S1).

2.2. Photophysical Properties. To understand the different dynamic optical properties of both polymorphs, the photophysical properties of $\mathrm{PyCz}$ phosphors in the crystal were investigated under ambient conditions. As shown in Figure 2(b), PyCz-B and PyCz-N both have weak phosphorescence signals in their initial states. After long photoactivation $\left(365 \mathrm{~nm}\right.$ light with the power of $40 \mathrm{~mW} / \mathrm{cm}^{2}$ for $10 \mathrm{~min}$ ), the phosphorescence intensity was largely enhanced by more than 6 times with little changes in emission peaks at 544 and $588 \mathrm{~nm}$, accompanied by a slight decrease in fluorescence intensity and lifetime (Figures 2(a) and S6 and Table S1). Simultaneously, the lifetimes of the emission bands at around $544 \mathrm{~nm}$ were prolonged from 44.52 to $868.86 \mathrm{~ms}$ and 20.24 to $776.03 \mathrm{~ms}$ for $\mathrm{PyCz}-\mathrm{B}$ and $\mathrm{PyCz}-\mathrm{N}$, respectively (Figures 2(c) and S7 and Table S2), suggesting photoactivated dynamic UOP behavior for both polymorphs. These dynamic UOP features were further confirmed by the prolonged emission lifetime (from 365.53 to $965.96 \mathrm{~ms}$ in PyCz-B and 208.50 to $1065.46 \mathrm{~ms}$ in $\mathrm{PyCz}-$ $\mathrm{N}$ ) in nitrogen during the long photoactivation (Figure S8 and Table S3). Such dynamic UOP behavior also occurred under the irradiation of weak UV light. As shown in Figure 2(d), the phosphorescence intensity enhanced slowly for both $\mathrm{PyCz}-\mathrm{B}$ and $\mathrm{PyCz}-\mathrm{N}$. The photoactivation took place within $20 \mathrm{~min}$ for PyCz-B and $>60 \mathrm{~min}$ for PyCz-N after irradiation by weak $365 \mathrm{~nm}$ light with excitation intensity of $0.67 \mathrm{~mW} / \mathrm{cm}^{2}$. The enhancement ratio $\left(I / I_{0}\right)$ of phosphorescence intensity was 3.7 and 3.1 for $\mathrm{PyCz}-\mathrm{B}$ and PyCz-N, respectively. In addition, this dynamic UOP behavior with different photoactivation speed was also observed by using excitation intensities of 0.56 and $0.39 \mathrm{~mW} / \mathrm{cm}^{2}$ (Figure S9 and Table S4). Notably, such a dynamic behavior of $\mathrm{PyCz}$ was reversible. The activated states of both polymorphs (PyCz-B(a) and $\mathrm{PyCz}-\mathrm{N}(\mathrm{a})$ ) can gradually return to their initial states (PyCz-B(i) and PyCz-N(i)) within a period of time when they were kept in the dark (Figure 2(e)), and such reversibility can be repeated for many times (Figure S10).

2.3. Experimental Investigation. To gain a deeper insight into the dynamic UOP behavior, the photophysical parameters of both polymorphs before and after photoactivation were calculated and tabulated in Table S10. It was found that the nonradiative decay rates in the activated state (1.15 and $\left.1.29 \mathrm{~s}^{-1}\right)$ are far less than those in the initial state (21.74 and $50.00 \mathrm{~s}^{-1}$ ) for both polymorphs (PyCz-B and PyCz-N,

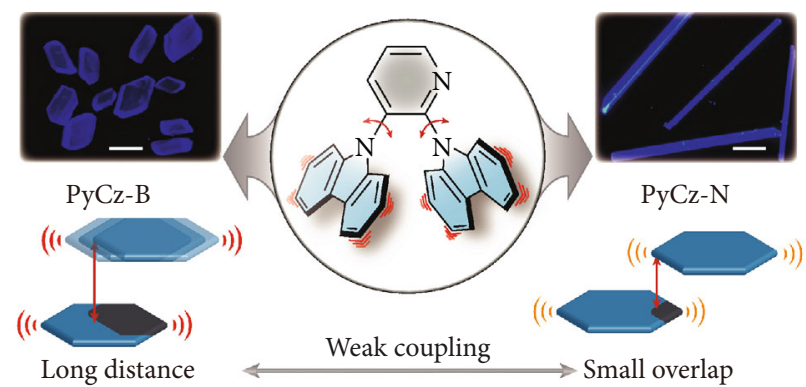

(a)

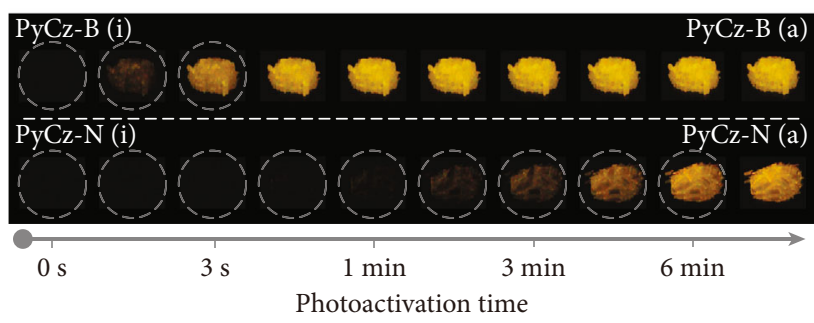

(b)

Figure 1: The structure of $\mathrm{PyCz}$ and two kinds of crystal of $\mathrm{PyCz}$ with different photoactivation UOP speed. (a) Molecular structure of $\mathrm{PyCz}$ with two kinds of molecular packing mode. Insets: the fluorescence microscopy images of PyCz-B and PyCz-N with the scale bar of $200 \mu \mathrm{m}$. (b) The afterglow photos of PyCz-B and $\mathrm{PyCz}-\mathrm{N}$ at different photoactivation time with a handheld $365 \mathrm{~nm}$ UV lamp $\left(40 \mathrm{~mW} / \mathrm{cm}^{2}\right)$ under ambient conditions, and the dashed circles are used to distinguish the samples with low afterglow intensity.

respectively), which are responsible for the prolonged UOP lifetime. Although, this decay rate is well-known to be related to quenching factor and molecular motions. Therefore, this is the first time we have studied the dynamic processes under different atmospheres to reveal the effect of the quenching factor from the external environment for two polymorphs (Figures 2(f), S11, and S12). With the alteration of atmosphere from oxygen to nitrogen, the lifetime $(\tau)$ for dynamic UOP behavior of both polymorphs have followed the same trend: the $\tau$ of photoactivation was shortened while it was prolonged for the deactivation process. Notably, $\mathrm{PyCz}-\mathrm{N}$ shows wider $\tau$ rangeability, indicating that $\mathrm{PyCz}-\mathrm{N}$ is more sensitive to the atmosphere. Besides, it is easily found that no matter how the atmosphere changed, the $\tau$ of $\mathrm{PyCz}-\mathrm{B}$ was always smaller than that of $\mathrm{PyCz}-\mathrm{N}$ under the same condition. So, we concluded that the atmosphere is not the cause for the different dynamic UOP feature of PyCz-B and $\mathrm{PyCz}-\mathrm{N}$.

When $\mathrm{PyCz}$ crystals were kept in liquid nitrogen environment $(77 \mathrm{~K})$, the dynamic UOP behavior was almost insensitive to the photoactivation time, while the deactivation process was prolonged. Especially, the deactivation time in PyCz-N at $77 \mathrm{~K}(>72 \mathrm{~h})$ is longer than that at $298 \mathrm{~K}(\sim 4 \mathrm{~h})$ (Figure S14). These phenomenon could be ascribed to the suppression of molecular motions. Furthermore, the singlecrystal analysis of both polymorphs was conducted before and after long photoactivation. In the initial state, the molecule in PyCz-N(i) was confined by multiple molecular 


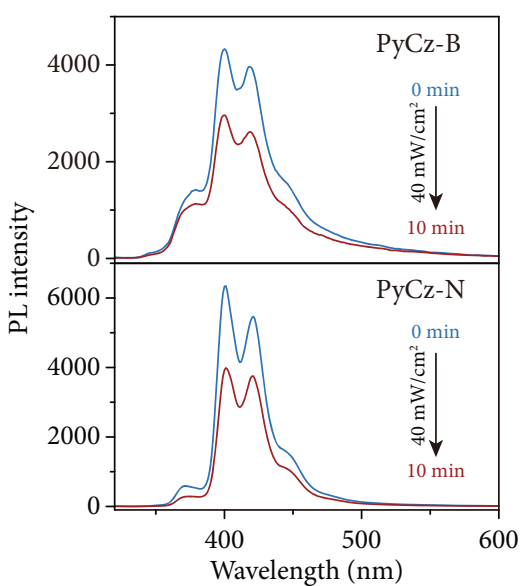

(a)

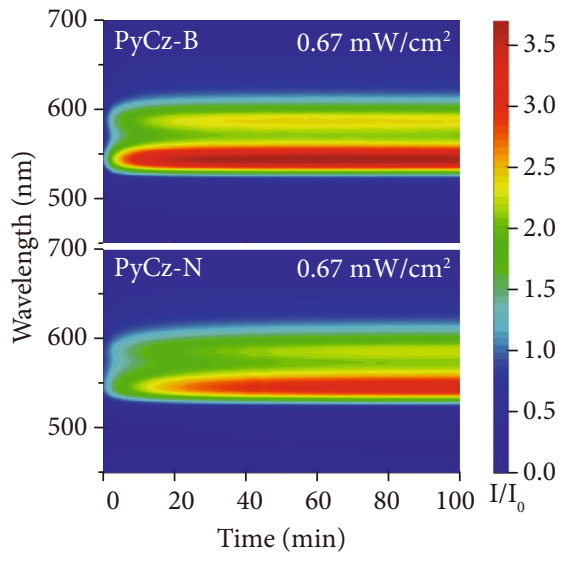

(d)

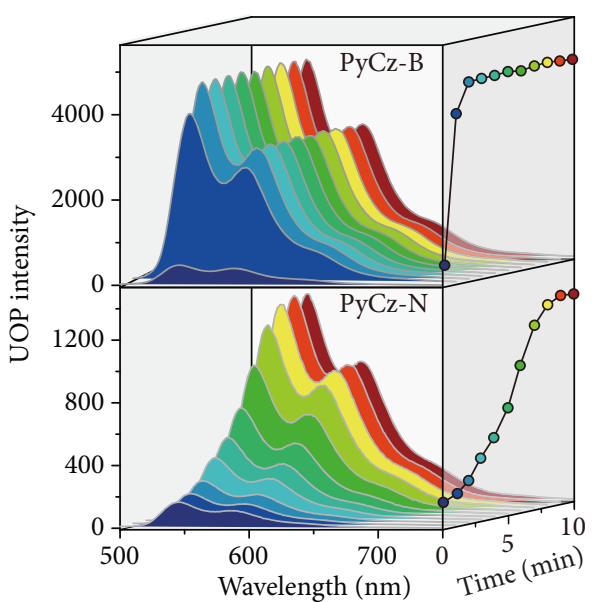

(b)

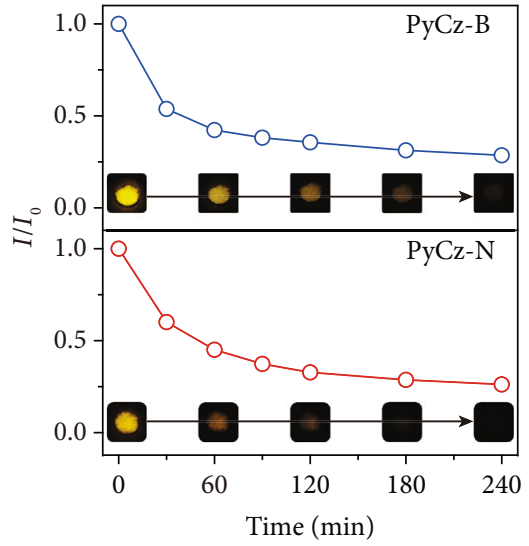

(e)

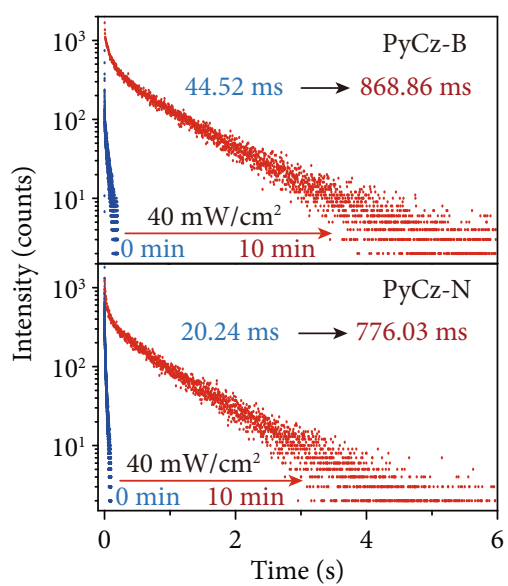

(c)

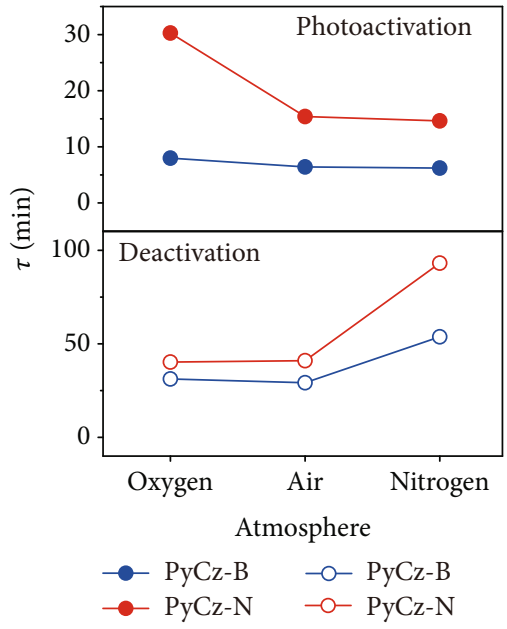

(f)

FIgure 2: Photophysical properties of PyCz under ambient conditions. (a) Steady-state PL spectra of PyCz-B and PyCz-N before (blue line) and after (red line) long photoactivation (power $=40 \mathrm{~mW} / \mathrm{cm}^{2}$, time $=10 \mathrm{~min}$ ) under ambient conditions. (b) The phosphorescence spectra and the corresponding peak intensity at $544 \mathrm{~nm}$ of $\mathrm{PyCz}-\mathrm{B}$ and $\mathrm{PyCz}-\mathrm{N}$ after different photoactivation time ranging from 0 to $10 \mathrm{~min}$ by a $365 \mathrm{~nm}$ lamp (power $=40 \mathrm{~mW} / \mathrm{cm}^{2}$ ) under ambient conditions. (c) Lifetime decay profiles of the emission peak at $544 \mathrm{~nm}$ of PyCz-N and $\mathrm{PyCz}-\mathrm{B}$ before and after long photoactivation under ambient conditions. (d) The time-phosphorescence mapping of PyCz-B and PyCz-N for $100 \mathrm{~min}$ with the $365 \mathrm{~nm}$ light source power of $0.67 \mathrm{~mW} / \mathrm{cm}^{2}$ under ambient conditions. (e) The phosphorescence variation value $\left(I / I_{0}\right)$ at $544 \mathrm{~nm}$ during the deactivation of the UOP for PyCz-N and PyCz-B after long photoactivation under ambient conditions. Insets: the corresponding photos at different deactivation time. (f) The corresponding dynamic lifetime $\tau$ for the photoactivation by weak $365 \mathrm{~nm}$ light (power $=0.67 \mathrm{~mW} / \mathrm{cm}^{2}$ ) and deactivation of $\mathrm{PyCz}-\mathrm{B}$ and $\mathrm{PyCz}-\mathrm{N}$ under different atmosphere.

interactions, including $\pi \cdots \pi(3.378 \AA$, $3.400 \AA), C-H \cdots \pi$ (2.701-2.873 $\AA$ ), and C-H...N (2.633 $\AA$ ) interactions, while there is only C-H $\cdots \pi(2.732-2.849 \AA)$ interactions in $\mathrm{PyCz}-$ $\mathrm{B}(\mathrm{i})$. After long photoactivation, obviously, molecular motions happened in the crystal and the distances of intermolecular interactions in $\mathrm{PyCz}-\mathrm{N}(\mathrm{a})$ decreased by 0.019-0.025 $\AA$, accompanied by newly formed $\pi \cdots \pi$ (3.388-3.399 $\AA)$ and $\mathrm{C}-\mathrm{H} \cdot \cdots \mathrm{N}(2.748 \AA)$ interactions. The decrease in intermolecular distances for $\mathrm{PyCz}-\mathrm{B}(\mathrm{a})$ is smaller (0.002-0.009 $\AA$ ) as compared to $\mathrm{PyCz}-\mathrm{N}(\mathrm{a})$, along with a new $\mathrm{C}-\mathrm{H} \cdots \pi(2.895 \AA)$ interaction formation (Figure 3(a) and Tables S8 and S9). With the enhancements of intermolecular interactions in both polymorphs, nonradiative transition could be suppressed, thus leading to longer emission lifetimes. Additionally, through the analysis of the independent gradient model (IGM) [53, 54], the molecular interactions could be directly displayed by the isosurface in Figure S16. After long photoactivation, the $\pi-\pi$ couplings and molecular interactions in both polymorphs have enhanced with larger isosurface, further proving that molecular motions have restricted for prolonging emission lifetimes after long photoactivation.

\subsection{Crystal Stacking Analysis and Simulated Calculations.} The molecular stacking may account for the different dynamic UOP behavior of $\mathrm{PyCz}$ polymorphs. As shown in Figure 3(b), both polymorphs before photoactivation have only weak $\pi-\pi$ couplings, which are not favorable for 


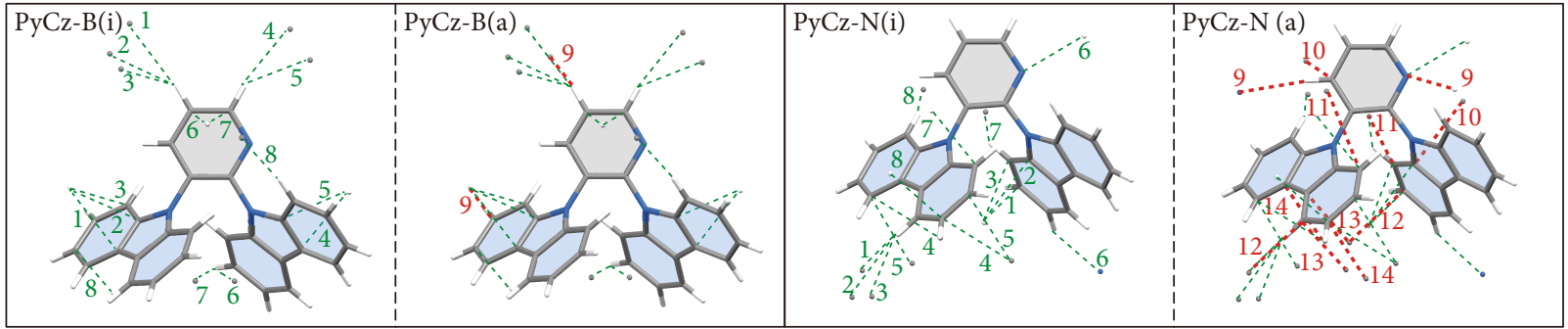

(a)

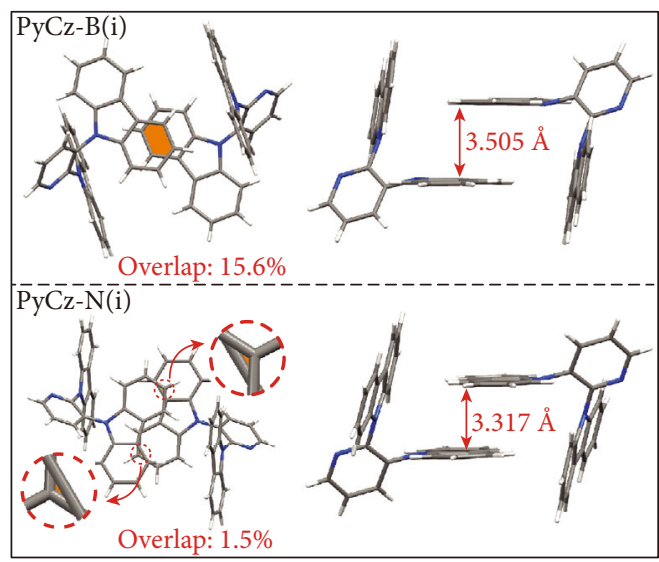

(b)

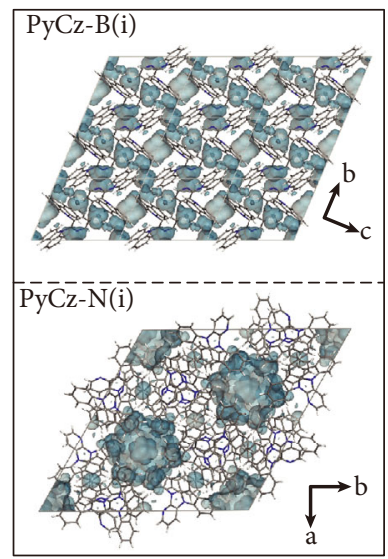

(c)

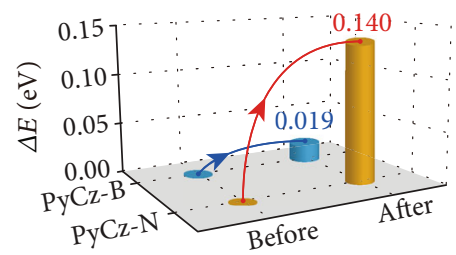

(d)

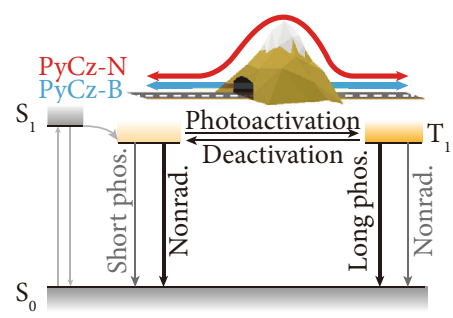

(e)

FIgURE 3: Crystal stacking analysis and simulated calculations for dynamic ultralong organic phosphorescence of PyCz. (a) The intermolecular interactions around one molecule in $\mathrm{PyCz}-\mathrm{B}$ and $\mathrm{PyCz}-\mathrm{N}$ before and after long photoactivation in crystalline state measured at $100 \mathrm{~K}$, the green dash line refers to the initial interactions and the red dash line is the added interactions after long photoactivation. (b) The $\pi-\pi$ overlap and distance of selected dimer with $\pi-\pi$ interactions in PyCz-B(i) and PyCz-N(i). Note that the green isosurface refer to the calculated molecular interactions by IGM, the isovalue is 0.008 . (c) The free volume region (cyan isosurface) in single crystal cells of PyCz-B(i) and PyCz-N(i). (d) The calculated change of single molecular energy in PyCz-B and PyCz-N during the process of photoactivation. (e) Proposed mechanism for different dynamic speeds of dynamic UOP in polymorphs.

phosphorescent emission [17, 42]. Specifically, the $\pi-\pi$ distance in PyCz-B (3.505 $\AA$ ) is larger than that in PyCz-N $(3.317 \AA)$, while the $\pi-\pi$ overlap in PyCz-N (1.5\%) is smaller than that in PyCz-B (15.6\%). Moreover, the isosurface with the same isovalue (0.008) in PyCz-N is much larger than that in $\mathrm{PyCz}-\mathrm{B}$, indicating the stronger intermolecular interactions. These results indicated that the weaker $\pi-\pi$ interactions caused by long $\pi-\pi$ distance may account for faster dynamic UOP in PyCz-B. To gain more insight into the interrelation between dynamic UOP and molecular stacking, a set of theoretical calculations was carried out. Firstly, the free volume distributions in $\mathrm{PyCz}-\mathrm{B}$ and $\mathrm{PyCz}-\mathrm{N}$ were calculated, which provides the space for molecular motions to rationally manipulate the nonradiative transitions during photoactivation. From Figure 3(c), it was found that the unoccupied spaces distributed more dispersedly around the molecules in PyCz-B, while in PyCz-N, the unoccupied spaces mostly concentrated in the cavities with a little space left around the molecules. Thus, molecules in $\mathrm{PyCz}-\mathrm{B}$ can adjust their configurations more easily and took less time to reach the activated states during photoactivation. Besides, the proportion of unoccupied spaces of $\mathrm{PyCz}-\mathrm{B}$ and $\mathrm{PyCz}-$ $\mathrm{N}$ become smaller after long photoactivation, from $12.19 \%$ to $12.06 \%$ and $16.86 \%$ to $16.40 \%$, respectively (Figure S17 and Table S11), further proving that the molecular stacking become tighter. In addition, the single molecular energy was calculated to characterize the energy variation from the initial state to the activated state. As illustrated in Figure 3(d), the energy increased by 0.019 and $0.140 \mathrm{eV}$ for PyCz-B and PyCz$\mathrm{N}$ after long photoactivation, respectively. In other words, there existed a larger energy barrier to overcome for PyCz-N molecules during the photoactivation. Owing to the smaller variation of single molecular energy, $\mathrm{PyCz}-\mathrm{B}$ can be activated or deactivated much more easily than $\mathrm{PyCz}-\mathrm{N}$, verifying the difference in dynamic performance from the energy aspect (Figure 3(e)). Taken together, we speculated that the different contribution of nonradiative transitions by manipulating different molecular stacking lead to distinct dynamic UOP features in $\mathrm{PyCz}$ polymorphs.

2.5. Applications. Nowadays, integration of multiple molecular logic gates to construct molecular computers is particularly challenging mainly due to connectivity of molecular logic gates. In optical devices, it is much harder to establish complex logic gates because the light signals were easily disturbed. Regarding the superiority of the ultralong 
emission lifetime for elimination of background fluorescence interference by time-resolved technique [55-57], we here attempted to utilize this dynamic UOP material for molecular logic gate (Figure 4(a)). From Figure 4(b), it could be easily found that the dynamic UOP property of $\mathrm{PyCz}$ polymorphs is highly dependent on crystal morphology and power of irradiation light source. With fixed power of UV light irradiation, the photoactivation speed of $\mathrm{PyCz}-\mathrm{B}$ was faster than that of PyCz-N under the same condition. Meanwhile, the stronger power of irradiation can greatly accelerate the photoactivation process. On the basis of abovementioned logic channels for rationally controlling the dynamic UOP, a molecular logic gate with two inputs and two outputs was fabricated. The crystal morphology (I1: PyCz-B is " 1 ", PyCz$\mathrm{N}$ is " 0 ") and the light power (I2: $40 \mathrm{~mW} / \mathrm{cm}^{2}$ is " 1 ", $0.67 \mathrm{~mW} / \mathrm{cm}^{2}$ is " 0 ") were defined as inputs, the relative intensity of phosphorescence $\left(I / I_{0}\right)$ at $1 \mathrm{~min}$ and $10 \mathrm{~min}$ was selected as output $1(\mathrm{O} 1)$ and output $2(\mathrm{O} 2)$, respectively. If the phosphorescent intensity $(I)$ is 3 times more than the initial intensity $\left(\mathrm{I}_{0}\right)$, the output is " 1 ." Otherwise, the value is "0." Thus, O1 and O2 can be recognized as the AND and OR phosphorescence-dependent molecular logic gate based on the truth table (Figure 4(c)). Additionally, with the reversibility of dynamic UOP (Figures S19 and S10), PyCz-N can also be used for rewritable timing data storage (Figure S20, Movie S3 and S4). The information can be stored for over 6 hours and can be rewritten by long photoactivation again. To the best of our knowledge, this is the first UOP example for molecular logic gate and rewritable timing data storage.

\section{Discussion}

In summary, we have developed two crystalline polymorphs of $\mathrm{PyCz}$ molecule, which showed distinct dynamic UOP behaviors owing to the different molecular stacking in crystal. PyCz-B showed much faster dynamic process than PyCz-N under the same conditions. Besides, it was found that the dynamic UOP behavior including photoactivation and deactivation features were highly dependent on irradiation intensity and environmental atmosphere for PyCz-B and PyCz-N. On the basis of experimental results and theoretical calculations, we speculated that the regulation of nonradiative transition through the manipulation of intermolecular stacking played a critical role in realizing various dynamic UOP behaviors. Given the fascinating optical features responding to multiple conditions, $\mathrm{PyCz}$ was successfully applied in phosphorescence-dependent molecular logic gate and timing data storage. This finding not only gives deeper understanding in photoactivatable dynamic UOP materials but also expands the scope of the applications of UOP materials.

\section{Materials and Methods}

4.1. Crystal Cultivation. Two types of crystals were prepared through slow solvent evaporation from different solutions. For $\mathrm{PyCz}-\mathrm{B}, 50 \mathrm{mg} \mathrm{PyCz}$ was refluxed and dissolved in $3 \mathrm{~mL}$ ethyl acetate; then, $2 \mathrm{~mL} n$-hexane was slowly injected over the solution; then, the solution was kept under ambient conditions; the block-like transparent crystal was incubated after four days. For PyCz-N, $50 \mathrm{mg}$ PyCz was dissolved in $3 \mathrm{~mL} \mathrm{CH}_{2} \mathrm{Cl}_{2}$; then, $2 \mathrm{~mL} n$-hexane was slowly injected over the solution; then, the solution was kept under ambient conditions; the needle-like transparent crystal was obtained after two days.

4.2. Measurements. Nuclear magnetic resonance $\left({ }^{1} \mathrm{H}\right.$ and ${ }^{13} \mathrm{C}$ NMR) spectra were obtained on a Bruker Ultra Shield plus $400 \mathrm{MHz}$ spectrometer. Chemical shift was relative to tetramethylsilane (TMS) as the internal standard. Resonance patterns were reported with the notation s (singlet), d (double), $\mathrm{t}$ (triplet), q (quartet), and m (multiplet). Mass spectra were obtained on a matrix-assisted laser desorption/ionization time of flight mass spectrometry (MALDI-TOF-MS). UV-visible absorption spectra were measured by Shimadzu UV-1750. Steady-state photoluminescence spectra, phosphorescence spectra, and excitation spectra were measured by using HitachiF-4600. The lifetime spectra were carried out on Edinburgh FLSP920 fluorescence spectrophotometer equipped with a xenon arc lamp (Xe900) or microsecond flash-lamp ( $\mu$ F900). Photoluminescence quantum efficiency was collected on a Hamamatsu Absolute PL Quantum Yield Spectrometer C11347 under ambient condition, the fluorescence and phosphorescence quantum efficiency $\left(\Phi_{\mathrm{F}}\right.$ and $\left.\Phi_{\mathrm{P}}\right)$ were calculated through the following formulas:

$$
\begin{aligned}
& \Phi_{P}=\Phi_{E} \times \frac{A_{P}}{A_{E}}, \\
& \Phi_{F}=\Phi_{E}-\Phi_{P},
\end{aligned}
$$

where $\Phi_{\mathrm{E}}$ refers to the measured total emission quantum efficiency, $A_{\mathrm{P}}$ and $A_{\mathrm{E}}$ refer to the integral areas of phosphorescence and photoluminescence components in photoluminescence spectra, respectively. During the measurements, the sample was firstly deposited into a quartz cuvette and put into the fluorescence spectrophotometer (HitachiF4600). Then, the cuvette with the phosphors was carefully fixed and irradiated by UV light with different power for a certain time. After the photoactivation process, the optical signal was collected. Luminescence photos and videos were taken by Cannon EOS 700D single lens digital cameras with a handheld UV lamp on and off. The fluorescent images of crystal were taken by Nikon DS-Ri2 Microscope Camera. The intensity of the UV lamp was measured by PA05-UVAB513-02 UV light meter.

X-ray crystallography was achieved by using a Bruker SMART APEX-II CCD diffractometer with graphite monochromated Mo-K $\alpha$ radiation. The structures of $\mathrm{PyCz}-\mathrm{B}$ and $\mathrm{PyCz}-\mathrm{N}$ before and after long photoactivation were measured in $100 \mathrm{~K}$ where the molecular motions would be restrained by low temperature. In order to exclude the influence of temperature factors in crystal, the crystal was kept in $100 \mathrm{~K}$ for over 10 minutes and then the measurement was started. After the first round of measurement, the crystal was photoactivated by a high-power UV lamp $\left(40 \mathrm{~mW} / \mathrm{cm}^{2}\right)$ for 10 minutes under room temperature and then the structure of crystal was measured as same as the previous round. Based on our observation, after the 


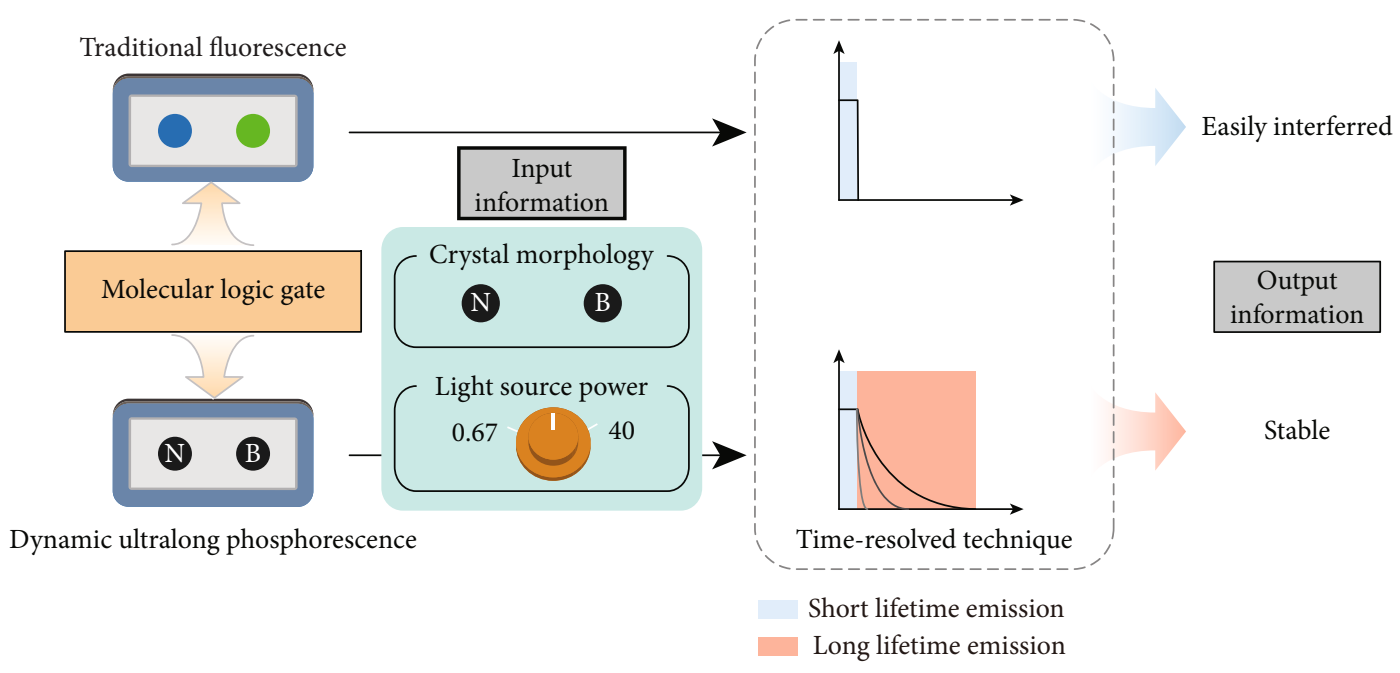

(a)

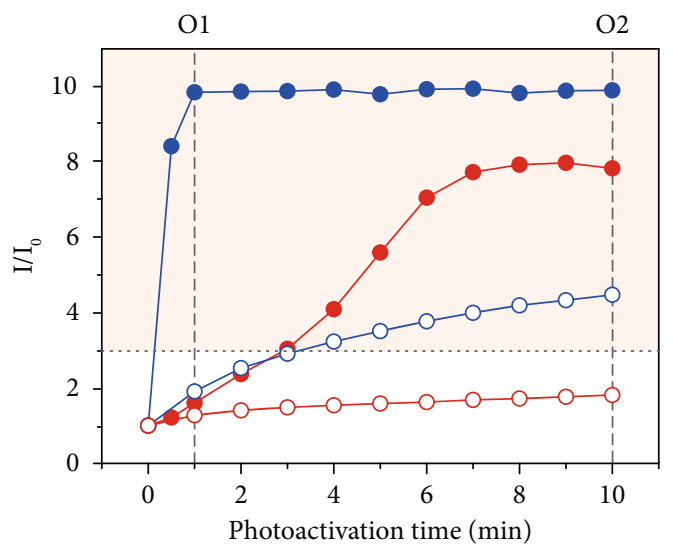

(b)

\begin{tabular}{ccccc}
\hline & I1 & I2 & O1 & O2 \\
\hline$\multimap$ & 1 & 1 & 1 & 1 \\
- & 0 & 1 & 0 & 1 \\
$\multimap-$ & 1 & 0 & 0 & 1 \\
- & 0 & 0 & 0 & 0 \\
\hline
\end{tabular}
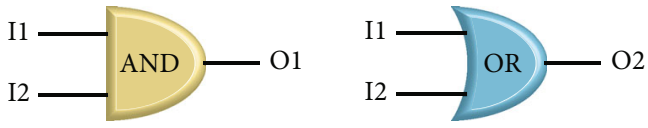

(c)

FIgURE 4: The demonstration of the application in molecular logic gate. (a) The model of molecular logic gate and the contrast between fluorescence logic gate and phosphorescence logic gate. (b) Under ambient conditions, the photoactivation of PyCz by different power UV light: PyCz-B activated by UV light with the power of $40 \mathrm{~mW} / \mathrm{cm}^{2}$ and $0.67 \mathrm{~mW} / \mathrm{cm}^{2}$ (blue line with solid and open circle, respectively), PyCz-N activated by UV light with the power of $40 \mathrm{~mW} / \mathrm{cm}^{2}$ and $0.67 \mathrm{~mW} / \mathrm{cm}^{2}$ (red line with solid and open circle, respectively). (c) The truth table for $\mathrm{O} 1$ and $\mathrm{O} 2$ and the proposed logic gate for $\mathrm{PyCz}$ with two inputs and two outputs.

second round of measurement, two kinds of crystal both still show long lifetime activated UOP thanks for the restriction of molecular motions by low temperature.

4.3. Computational Details. All theoretical calculations in this work were based on the measured crystal data of PyCz-B and $\mathrm{PyCz}-\mathrm{N}$ before and after long photoactivation. The analysis of the independent gradient model (IGM) was carried out by Multiwfn 3.6 [53] and volume was rendered by VMD 1.9.3 [54] based on the selected molecular dimers in the crystal data of $\mathrm{PyCz}-\mathrm{B}$ and $\mathrm{PyCz}-\mathrm{N}$ before and after long photoactivation. The isovalue can be set in VMD 1.9.3 to adjust the scale of isosurface. The free volume distributions were calculated using Materials Studio [58] software with $0.2 \AA$ diameter sensor based on the selected crystal cells of $\mathrm{PyCz}-\mathrm{B}$ and $\mathrm{PyCz}-\mathrm{N}$ before and after long photoactivation. The single molecular energy was evaluated at B3LYP/6-
31G(d) level by Gaussian 09 program based on the one molecular configuration in the crystal data [59].

\section{Conflicts of Interest}

The authors declare that there is no conflict of interest regarding the publication of this article.

\section{Authors' Contributions}

Z. An and W. Huang conceived the projects. M. Gu conducted the experiments. K. Ling and K. Huang grew the crystals and measured the single crystal structures. $\mathrm{H}$. Ma and A. Lv. performed the theoretical calculations. H. Wang and L. Gu measured the lifetime. M. Gu, H. Ma, M. Singn, W. Yao, H. Shi, Z. An and W. Huang analyzed the data, wrote and revised the manuscript. All authors discussed the results 
and commented on the manuscript. Mingxing $\mathrm{Gu}$ and Huifang Shi contributed equally to this work.

\section{Acknowledgments}

This work is supported by the National Natural Science Foundation of China (21875104, 21975120, 21973043, 51673095, and 91833302) and Natural Science Fund for Distinguished Young Scholars of Jiangsu Province (BK20180037). We are grateful to the High Performance Computing Center of Nanjing Tech University for supporting the computational resources.

\section{Supplementary Materials}

Supplementary 1. Table S1: fluorescence lifetimes $(\tau \mathrm{f})$ of $\mathrm{PyCz}-\mathrm{B}$ and $\mathrm{PyCz}-\mathrm{N}$ before and after long photoactivation under ambient conditions. Table S2: ultralong organic phosphorescence lifetimes ( $\tau$ p) of PyCz-B and PyCz-N before and after long photoactivation under ambient conditions. Table S3: ultralong organic phosphorescence lifetimes $(\tau \mathrm{p})$ of $\mathrm{PyCz}-\mathrm{B}$ and $\mathrm{PyCz}-\mathrm{N}$ before and after long photoactivation under nitrogen. Table S4: the fitting results of the photoactivation process of $\mathrm{PyCz}-\mathrm{B}$ and $\mathrm{PyCz}-\mathrm{N}$ activated by UV light with different excitation intensity. Table S5: the fitting result of the process of photoactivation and deactivation of PyCz-B and PyCz-N under different atmospheres in Figure S11 and Figure S12. Table S6: the photoluminescent quantum yield (\%) of PyCz-N and $\mathrm{PyCz}-\mathrm{B}$ before and after long photoactivation under ambient conditions. Table S7: structure data of $\mathrm{PyCz}-\mathrm{N}$ and $\mathrm{PyCz}-\mathrm{B}$ before and after long photoactivation for 10 minutes at $100 \mathrm{~K}$. Table S8: the intermolecular interactions of $\mathrm{PyCz}-\mathrm{B}$ before and after long photoactivation. Table S9: the intermolecular interactions of $\mathrm{PyCz}-\mathrm{N}$ before and after long photoactivation. Table S10: the photophysical parameters of the dynamic UOP phosphors before and after long photoactivation. Table S11. Calculated occupied volume, free volume, and the proportion of free volume in $\mathrm{PyCz}-\mathrm{B}$ and $\mathrm{PyCz}-\mathrm{N}$ before and after long photoactivation. Figure $\mathrm{S} 1 \mathrm{l} \mathrm{H}$ NMR spectrum of PyCz in DMSO. Figure S2: 13C NMR spectrum of PyCz in DMSO. Figure S3: MALDI-TOF spectrum of $\mathrm{PyCz}$ molecule. Figure S4: photophysical properties of $\mathrm{PyCz}$ molecule in dilute 2-methyltetrahydrofuran $\left(1 \times 10^{-5} \mathrm{M}\right)$. Figure S5: excitation spectra monitoring the UOP emission band at $544 \mathrm{~nm}$ for $\mathrm{PyCz}-\mathrm{B}$ and $\mathrm{PyCz}-\mathrm{N}$ before and after long photoactivation. Figure S6: lifetime decay curves of the fluorescence emission bands of $\mathrm{PyCz}-\mathrm{B}$ and $\mathrm{PyCz}-\mathrm{N}$ before and after long photoactivation under ambient conditions. Figure S7: lifetime decay curves of the phosphorescence emission bands of PyCz-B and PyCz-N before and after long photoactivation under ambient conditions. Figure S8: lifetime decay curves of the phosphorescence emission bands of PyCz-B and $\mathrm{PyCz}-\mathrm{N}$ before and after long photoactivation under nitrogen. Figure S9: the phosphorescence intensity at $544 \mathrm{~nm}$ of PyCz-B and PyCz-N after different photoactivation time by a $365 \mathrm{~nm}$ lamp (power $=0.67,0.56$, and $0.39 \mathrm{~mW} / \mathrm{cm} 2$ ) under ambient conditions. Figure S10: reversible photoactivation and deactivation cycles of $\mathrm{PyCz}-\mathrm{B}$ and PyCz-N under ambient conditions. Figure S11: the dynamic
UOP properties of $\mathrm{PyCz}$ crystals under different atmospheres. Figure S12: the deactivated properties of dynamic UOP for $\mathrm{PyCz}$ crystal under different atmospheres. Figure S13: phosphorescence spectra of $\mathrm{PyCz}-\mathrm{N}$ under different temperatures ranging from 173 to $273 \mathrm{~K}$. The corresponding photographs of PyCz-N under daylight, UV on, and UV off at 273, 253, 233, 213, 193, and $77 \mathrm{~K}$. Figure S14: the deactivation process of PyCz-N in $77 \mathrm{~K}$. Figure S15: the angles between triazine and carbazole units of PyCz in optimized S0, S1, and T1 geometry in gas phases. Figure S16: the calculated molecular interactions in dimers of $\mathrm{PyCz}-\mathrm{B}(\mathrm{i}), \mathrm{PyCz}-\mathrm{B}, \mathrm{PyCz}-\mathrm{N}(\mathrm{i})$, and $\mathrm{PyCz}-\mathrm{N}(\mathrm{a})$. Figure S17: free volume region in single crystal cells of $\mathrm{PyCz}-\mathrm{B}$ and $\mathrm{PyCz}-\mathrm{N}$ after long photoactivation. Figure S1: the cavities in PyCz-N before and after long photoactivation. Figure S19: the relative intensity $\left(I / I_{0}\right)$ of $544 \mathrm{~nm}$ UOP of $\mathrm{PyCz}-\mathrm{N}$ as a function of long photoactivation and deactivation time. Figure S20: the application of $\mathrm{PyCz}$ for rewritable timing data storage.

Supplementary 2. Movie S1: the process of long photoactivation of $\mathrm{PyCz}-\mathrm{B}$ and $\mathrm{PyCz}-\mathrm{N}$ under the irradiation of a strong UV lamp with the power of $40 \mathrm{~mW} / \mathrm{cm} 2$.

Supplementary 3. Movie S2: the afterglow of PyCz-N under different temperature.

Supplementary 4. Movie S3: the writing process of the rewritable visual data storage based on $\mathrm{PyCz}-\mathrm{N}$ phosphor.

Supplementary 5. Movie S4: the erasing process of the rewritable visual data storage based on $\mathrm{PyCz}-\mathrm{N}$ phosphor.

\section{References}

[1] O. Sato, "Dynamic molecular crystals with switchable physical properties," Nature Chemistry, vol. 8, no. 7, pp. 644656, 2016.

[2] M. A. C. Stuart, W. T. S. Huck, J. Genzer et al., "Emerging applications of stimuli-responsive polymer materials," Nature Materials, vol. 9, no. 2, pp. 101-113, 2010.

[3] Y. Wang, K. Zhou, G. Huang et al., "A nanoparticle-based strategy for the imaging of a broad range of tumours by nonlinear amplification of microenvironment signals," Nature Materials, vol. 13, no. 2, pp. 204-212, 2014.

[4] S. Chen, Y. Hong, Y. Liu et al., "Full-range intracellular $\mathrm{pH}$ sensing by an aggregation-induced emission-active twochannel ratiometric fluorogen," Journal of the American Chemical Society, vol. 135, no. 13, pp. 4926-4929, 2013.

[5] X. Ji, Y. Yao, J. Li, X. Yan, and F. Huang, "A supramolecular cross-linked conjugated polymer network for multiple fluorescent sensing," Journal of the American Chemical Society, vol. 135, no. 1, pp. 74-77, 2013.

[6] X. Zhu, R. Liu, Y. Li et al., "An AIE-active boron-difluoride complex: multi-stimuli-responsive fluorescence and application in data security protection," Chemical Communications, vol. 50, no. 85, pp. 12951-12954, 2014.

[7] X. Hou, C. Ke, C. J. Bruns, P. R. McGonigal, R. B. Pettman, and J. F. Stoddart, "Tunable solid-state fluorescent materials for supramolecular encryption," Nature Communications, vol. 6, no. 1, pp. 6884-6892, 2015.

[8] J. W. Chung, S.-J. Yoon, S.-J. Lim, B.-K. An, and S. Y. Park, "Dual-mode switching in highly fluorescent organogels: binary 
logic gates with optical/thermal inputs," Angewandte Chemie International Edition, vol. 48, no. 38, pp. 7030-7034, 2009.

[9] D. Genovese, A. Aliprandi, E. A. Prasetyanto et al., "Mechanoand photochromism from bulk to nanoscale: data storage on individual self-assembled ribbons," Advanced Functional Materials, vol. 26, no. 29, pp. 5271-5278, 2016.

[10] D. Margulies, C. E. Felder, G. Melman, and A. Shanzer, "A molecular keypad lock: a photochemical device capable of authorizing password entries," Journal of the American Chemical Society, vol. 129, no. 2, pp. 347-354, 2007.

[11] S. Erbas-Cakmak, S. Kolemen, A. C. Sedgwick et al., "Molecular logic gates: the past, present and future," Chemical Society Reviews, vol. 47, no. 7, pp. 2228-2248, 2018.

[12] S. Xu, R. Chen, C. Zheng, and W. Huang, "Excited state modulation for organic afterglow: materials and applications," Advanced Materials, vol. 28, no. 45, pp. 9920-9940, 2016.

[13] N. Gan, H. Shi, Z. An, and W. Huang, "Recent advances in polymer-based metal-free room-temperature phosphorescent materials," Advanced Functional Materials, vol. 28, no. 51, article 1802657, 2018.

[14] S. Hirata, "Recent advances in materials with roomtemperature phosphorescence: photophysics for triplet exciton stabilization," Advanced Optical Materials, vol. 5, no. 17, article 1700116, 2017.

[15] Kenry, C. Chen, and B. Liu, "Enhancing the performance of pure organic room-temperature phosphorescent luminophores," Nature Communications, vol. 10, no. 1, p. 2111, 2019.

[16] Z. He, W. Zhao, J. W. Y. Lam et al., "White light emission from a single organic molecule with dual phosphorescence at room temperature," Nature Communications, vol. 8, no. 1, pp. 416423, 2017.

[17] Z. Chai, C. Wang, J. Wang et al., “Abnormal room temperature phosphorescence of purely organic boron-containing compounds: the relationship between the emissive behaviorand the molecular packing, and the potential related applications," Chemical Science, vol. 8, no. 12, pp. 8336-8344, 2017.

[18] Y. Shoji, Y. Ikabata, Q. Wang et al., "Unveiling a new aspect of simple arylboronic esters: long-lived room-temperature phosphorescence from heavy-atom-free molecules," Journal of the American Chemical Society, vol. 139, no. 7, pp. 2728-2733, 2017.

[19] W. Zhao, Z. He, J. W. Y. Lam et al., "Rational molecular design for achieving persistent and efficient pure organic roomtemperature phosphorescence," Chem, vol. 1, no. 4, pp. 592602, 2016.

[20] N. Gan, X. Wang, H. Ma et al., "Manipulating the stacking of triplet chromophores in the crystal form for ultralong organic phosphorescence," Angewandte Chemie International Edition, vol. 58, no. 40, pp. 14140-14145, 2019.

[21] Z. He, H. Gao, S. Zhang et al., "Achieving persistent, efficient, and robust room-temperature phosphorescence from pure organics for versatile applications," Advanced Materials, vol. 31, no. 18, article 1807222, 2019.

[22] K. Narushima, Y. Kiyota, T. Mori, S. Hirata, and M. Vacha, "Suppressed triplet exciton diffusion due to small orbital overlap as a key design factor for ultralong-lived roomtemperature phosphorescence in molecular crystals," Advanced Materials, vol. 31, no. 10, article 1807268, 2019.

[23] K. Ling, H. Shi, H. Wang et al., "Controllable multiemission with ultralong organic phosphorescence in crystal by isomeri- zation," Advanced Optical Materials, vol. 7, no. 24, article 1901076, 2019.

[24] Z. An, C. Zheng, Y. Tao et al., "Stabilizing triplet excited states for ultralong organic phosphorescence," Nature Materials, vol. 14, no. 7, pp. 685-690, 2015.

[25] S. Cai, H. Shi, J. Li et al., "Visible-light-excited ultralong organic phosphorescence by manipulating intermolecular interactions," Advanced Materials, vol. 29, no. 35, article $1701244,2017$.

[26] S. Cai, H. Shi, Z. Zhang et al., "Hydrogen-bonded organic aromatic frameworks for ultralong phosphorescence by intralayer $\pi-\pi$ interactions," Angewandte Chemie International Edition, vol. 57, no. 15, pp. 4005-4009, 2018.

[27] E. Lucenti, A. Forni, C. Botta et al., "Cyclic triimidazole derivatives: intriguing examples of multiple emissions and ultralong phosphorescence at room temperature," Angewandte Chemie International Edition, vol. 56, no. 51, pp. 16302-16307, 2017.

[28] L. Gu, H. Shi, L. Bian et al., "Colour-tunable ultra-long organic phosphorescence of a single-component molecular crystal," Nature Photonics, vol. 13, no. 6, pp. 406-411, 2019.

[29] S. Hirata, K. Totani, J. Zhang et al., "Efficient persistent room temperature phosphorescence in organic amorphous materials under ambient conditions," Advanced Functional Materials, vol. 23, no. 27, pp. 3386-3397, 2013.

[30] J. Wei, B. Liang, R. Duan et al., "Induction of strong long-lived room-temperature phosphorescence of $\mathrm{N}$-phenyl-2-naphthylamine molecules by confinement in a crystalline dibromobiphenyl matrix," Angewandte Chemie International Edition, vol. 55, no. 50, pp. 15589-15593, 2016.

[31] O. Bolton, K. Lee, H. J. Kim, K. Y. Lin, and J. Kim, “Activating efficient phosphorescence from purely organic materials by crystal design," Nature Chemistry, vol. 3, no. 3, pp. 205-210, 2011.

[32] D. Lee, O. Bolton, B. C. Kim, J. H. Youk, S. Takayama, and J. Kim, "Room temperature phosphorescence of metal-free organic materials in amorphous polymer matrices," Journal of the American Chemical Society, vol. 135, no. 16, pp. 63256329, 2013.

[33] L. Bian, H. Shi, X. Wang et al., "Simultaneously enhancing efficiency and lifetime of ultralong organic phosphorescence materials by molecular self-assembly," Journal of the American Chemical Society, vol. 140, no. 34, pp. 10734-10739, 2018.

[34] Z. Cheng, H. Shi, H. Ma et al., "Ultralong phosphorescence from organic ionic crystals under ambient conditions," Angewandte Chemie International Edition, vol. 57, no. 3, pp. 678682, 2018.

[35] Z. Yang, Z. Mao, X. Zhang et al., "Intermolecular electronic coupling of organic units for efficient persistent roomtemperature phosphorescence," Angewandte Chemie International Edition, vol. 55, no. 6, pp. 2181-2185, 2016.

[36] H. Hu, F. Meier, D. Zhao et al., "Efficient room-temperature phosphorescence from organic-inorganic hybrid perovskites by molecular engineering," Advanced Materials, vol. 30, no. 36, article 1707621, 2018.

[37] Q. Li, M. Zhou, M. Yang, Q. Yang, Z. Zhang, and J. Shi, "Induction of long-lived room temperature phosphorescence of carbon dots by water in hydrogen-bonded matrices," Nature Communications, vol. 9, no. 1, pp. 734-741, 2018.

[38] X. Chen, C. Xu, T. Wang et al., "Versatile room-temperaturephosphorescent materials prepared from $\mathrm{N}$-substituted naphthalimides: emission enhancement and chemical 
conjugation," Angewandte Chemie International Edition, vol. 55, no. 34, pp. 9872-9876, 2016.

[39] X. Yang and D. Yan, "Long-afterglow metal-organic frameworks: reversible guest-induced phosphorescence tunability," Chemical Science, vol. 7, no. 7, pp. 4519-4526, 2016.

[40] Y. Tao, R. Chen, H. Li et al., "Resonance-activated spinflipping for efficient organic ultralong room-temperature phosphorescence," Advanced Materials, vol. 30, no. 44, article 1803856, 2018.

[41] L. Xiao, Y. Wu, Z. Yu et al., "Room-temperature phosphorescence in pure organic materials: halogen bonding switching effects," Chemistry - A European Journal, vol. 24, no. 8, pp. 1801-1805, 2018.

[42] M. S. Kwon, Y. Yu, C. Coburn et al., "Suppressing molecular motions for enhanced room-temperature phosphorescence of metal-free organic materials," Nature Communications, vol. 6, no. 1, pp. 8947-8955, 2015.

[43] S. Cai, H. Ma, H. Shi et al., "Enabling long-lived organic room temperature phosphorescence in polymers by subunit interlocking," Nature Communications, vol. 10, no. 1, p. 4247, 2019.

[44] H. Wang, H. Shi, W. Ye et al., "Amorphous ionic polymers with color-tunable ultralong organic phosphorescence," Angewandte Chemie International Edition, vol. 58, no. 52, pp. 18776-18782, 2019.

[45] L. Gu, H. Shi, M. Gu et al., "Dynamic ultralong organic phosphorescence by photoactivation," Angewandte Chemie International Edition, vol. 57, no. 28, pp. 8425-8431, 2018.

[46] J. Yang, X. Zhen, B. Wang et al., “The influence of the molecular packing on the room temperature phosphorescence of purely organic luminogens," Nature Communications, vol. 9, no. 1, pp. 840-850, 2018.

[47] X. Jia, C. Shao, X. Bai et al., "Photoexcitation-controlled selfrecoverable molecular aggregation for flicker phosphorescence," Proceedings of the National Academy of Sciences of the United States of America, vol. 116, no. 11, pp. 4816-4821, 2019.

[48] Q. Huang, X. Mei, Z. Xie et al., "Photo-induced phosphorescence and mechanoluminescence switching in a simple purely organic molecule," Journal of Materials Chemistry C, vol. 7, no. 9, pp. 2530-2534, 2019.

[49] D. Yan and D. G. Evans, "Molecular crystalline materials with tunable luminescent properties: from polymorphs to multicomponent solids," Materials Horizons, vol. 1, no. 1, pp. 4657, 2014

[50] Y. Xie, Y. Ge, Q. Peng, C. Li, Q. Li, and Z. Li, "How the molecular packing affects the room temperature phosphorescence in pure organic compounds: ingenious molecular design, detailed crystal analysis, and rational theoretical calculations," Advanced Materials, vol. 29, no. 17, article 1606829, 2017.

[51] J. Yang, Z. Ren, B. Chen et al., "Three polymorphs of one luminogen: how the molecular packing affects the RTP and AIE properties?," Journal of Materials Chemistry C, vol. 5, no. 36, pp. 9242-9246, 2017.

[52] J. Wang, C. Wang, Y. Gong et al., "Bromine-substituted fluorene: molecular structure, $\mathrm{Br}-\mathrm{Br}$ interactions, roomtemperature phosphorescence, and tricolor triboluminescence," Angewandte Chemie International Edition, vol. 57, no. 51, pp. 16821-16826, 2018.

[53] T. Lu and F. Chen, "Multiwfn: a multifunctional wavefunction analyzer," Journal of Computational Chemistry, vol. 33, no. 5, pp. 580-592, 2012.
[54] W. Humphrey, A. Dalke, and K. Schulten, "VMD: visual molecular dynamics," Journal of Molecular Graphics \& Modelling, vol. 14, no. 1, pp. 33-38, 1996.

[55] A. P. De Silva, Molecular Logic-Based Computation, Royal Society of Chemistry, Cambridge, UK, 2013.

[56] K. Y. Zhang, Q. Yu, H. Wei, S. Liu, Q. Zhao, and W. Huang, "Long-lived emissive probes for time-resolved photoluminescence bioimaging and biosensing," Chemical Reviews, vol. 118, no. 4, pp. 1770-1839, 2018.

[57] H. Sun, S. Liu, W. Lin et al., "Smart responsive phosphorescent materials for data recording and security protection," Nature Communications, vol. 5, no. 1, p. 3601, 2014.

[58] Material Studio, version 7.0, BIOVIA, San Diego, CA, 2013, https://www.materials-studio.com.

[59] M. J. Frisch, G. W. Trucks, H. B. Schlegel et al., Gaussian 09. Revision C.01., Gaussian, Inc., Wallingford, CT, USA, 2009. 